\title{
New Therapies for Diabetes Management
}

\author{
Satish K. Garg
}

\section{Introduction}

D ESPITE ALL OF THE ADVANCES in the availability of new drugs and devices in the past two decades, the diabetes treatment outcome continues to be suboptimal. Part of the reason for not achieving target $\mathrm{A} 1 \mathrm{c}$ in subjects with diabetes may be due to improper timing and implementation of these drugs and devices in day-to-day management. In this chapter, we review current therapies (insulin and oral medications) for management of type 1 and type 2 diabetes. We are not going to address any of the devices, and some of the insulin treatments, in this chapter as they have been previously discussed in other chapters on the use of CGM, insulin pump, and insulin treatment options.

\section{SGLT2 inhibitors: a new emerging therapeutic class in the treatment of type 2 diabetes mellitus}

\section{Ghosh $R K^{1}$, Ghosh $S M^{2}$, Chawla $S^{3}$, Jasdanwala $S A^{1}$}

${ }^{1}$ Maulana Azad Medical College, New Delhi, India; ${ }^{2}$ Lady Hardinge Medical College, New Delhi, India; and ${ }^{3}$ University of Delhi, New Delhi, India

Clin Pharmacol 2012; 52: 457-63

The global incidence of type 2 diabetes mellitus continues to increase. Existing therapeutic classes of antidiabetic drugs are not adequate for maintaining long-term glycemic control in most patients, even when used in combination. Sodium glucose cotransporter 2 (SGLT2) inhibitors represent a promising new therapeutic class of antidiabetic drugs. SGLT2 accounts for $90 \%$ of glucose reabsorption in the kidney. SGLT2 inhibitors increase urinary excretion of glucose, resulting in lower plasma glucose concentrations independent of insulin levels. Dapagliflozin is the lead molecule in development in this class. It is currently in a phase III clinical trial. Other members of this class (e.g., sergliflozin and remogliflozin) are in various stages of clinical testing. This class of novel agents can effectively control blood sugar level without producing weight gain or hypoglycemia. Results of ongoing phase III clinical trials will be critical for determining whether the risk-benefit ratio will support the approval of this new class of drugs for the management of type 2 diabetes mellitus.

\section{A novel series of glucagon receptor antagonists with reduced molecular weight and lipophilicity}

Filipski KJ, Bian J, Ebner DC, Lee EC, Li JC, Sammons MF, Wright SW, Stevens BD, Didiuk MT, Tu M, Perreault C, Brown J, Atkinson K, Tan B, Salatto CT, Litchfield J, Pfefferkorn JA, Guzman-Perez A

Pfizer Worldwide Research E Development, Groton, CT

Bioorg Med Chem Lett 2012; 22: 415-20

A novel series of glucagon receptor antagonists with better druglike properties than existing molecules has been discovered. These pyrazole ethers and aminopyrazoles have lower molecular weight and increased polarity. The work presented here describes two new glucagon receptor antagonists, compounds 44 and 50, which were shown to have good pharmacokinetic properties in dogs, compared to rats, in which clearance was high. This study also evaluated compound 49, which demonstrated a dose-dependent reduction in glucose excursion in a rat glucagon challenge experiment.

\section{Linagliptin: the newest dipeptidyl peptidase-4 inhibitor for type 2 diabetes mellitus}

\section{Aletti R, Cheng-Lai A}

Department of Pharmacy, Montefiore Medical Center/Jack D. Weiler Hospital of the Albert Einstein College of Medicine, Bronx, NY

Cardiol Rev 2012; 20: 45-51

Dipeptidyl peptidase-4 (DPP-4) inhibitors are some of the newest medications used in the management of patients with type 2 diabetes mellitus. By inhibiting DPP- 4 enzyme, these compounds increase the amount of circulating incretin hormones, leading to greater insulin release and suppression of glucagon secretion. Linagliptin is the third DPP-4 inhibitor approved by the U.S. Food and Drug Administration. It has

Barbara Davis Center for Childhood Diabetes, University of Colorado Health Sciences Center, Aurora, CO 
the potential for use as monotherapy or as an adjunctive therapy in dual or triple combination regimens with other oral agents. Linagliptin results in a decrease in glycosylated hemoglobin by about $0.4 \%$ when used as monotherapy and by about $0.5 \%$ to $1.1 \%$ when used in combination with other oral antihyperglycemic agents. (The body excretes about $80 \%$ of linagliptin via the enterohepatic system.) Since only a minimal amount is eliminated through renal excretion, dosage adjustment is not necessary in patients with renal impairment. Another advantage of linagliptin is its favorable safety profile, with nasopharyngitis being one of the more commonly observed side effects. The promising safety and efficacy profiles of linagliptin make it a good alternative to the other two agents in this class, especially for patients with renal impairment. This article reviews the pharmacologic and pharmacokinetic properties of linagliptin. Key differences among the three available DPP-4 inhibitors are also examined.

\section{Empagliflozin, a novel selective sodium glucose cotransporter-2 (SGLT-2) inhibitor: characterisation and comparison with other SGLT-2 inhibitors}

Grempler $R^{1}$, Thomas $L^{1}$, Eckhardt $M^{2}$, Himmelsbach $F^{2}$, Sauer $A^{3}$, Sharp $D E^{4}$, Bakker $R A^{1}$, Mark $M^{1}$, Klein $T^{1}$, Eickelmann $P^{1}$

${ }^{1}$ CardioMetabolic Diseases Research, Boehringer Ingelheim Pharma GmbH \& Co.KG, Biberach, Germany; ${ }^{2}$ Medicinal Chemistry, Boehringer Ingelheim Pharma GmbH \& Co.KG, Biberach, Germany; ${ }^{3}$ Drug Discovery Support, Boehringer Ingelheim Pharma GmbH E Co.KG, Biberach, Germany; and ${ }^{4}$ Drug Metabolism and Pharmacokinetics, Boehringer Ingelheim Pharmaceuticals Inc., Ridgefield, CT

Diabetes Obes Metab 2012; 14: 83-90

\section{Objectives}

Empagliflozin is a selective sodium glucose cotransporter-2 (SGLT-2) inhibitor in clinical testing for the treatment of patients with type 2 diabetes mellitus. The purpose of this study was to assess the pharmacological properties of empagliflozin in vitro as well as its pharmacokinetic properties in vivo and to compare the drug's potency and selectivity with other SGLT-2 inhibitors.

\section{Methods}

[(14)C]-alpha-methyl glucopyranoside (AMG) uptake experiments were performed in stable cell lines over-expressing human (h)SGLT-1, 2, and 4. Two new cell lines overexpressing hSGLT-5 and hSGLT-6 were established, and [(14)C]-mannose and [(14)C]-myo-inositol uptake assays were developed. To analyze binding kinetics, a radioligand binding assay with [(3)H]-labeled empagliflozin and HEK293-hSGLT-2 cell membranes was used. Tests to assess acute in vivo pharmacokinetics were performed in normoglycemic beagle dogs and Zucker diabetic fatty (ZDF) rats.

\section{Results}

Empagliflozin has an IC(50) of $3.1 \mathrm{nM}$ for hSGLT-2. Binding of empagliflozin to SGLT-2 is competitive with glucose (halflife approximately $1 \mathrm{~h}$ ). Compared with other SGLT-2 inhibitors, empagliflozin has a high degree of selectivity over
SGLT-1, 4, 5, and 6. Species differences in SGLT-1 selectivity were identified. Pharmacokinetic studies in ZDF rats demonstrated moderate total plasma clearance (CL) and bioavailability (BA), while in beagle dogs CL was low and BA was high.

\section{Conclusions}

Empagliflozin is a potent and competitive SGLT-2 inhibitor with an excellent selectivity profile and the highest selectivity window of the tested SGLT-2 inhibitors over hSGLT-1. Empagliflozin represents an innovative therapeutic option for the treatment of patients with diabetes.

\section{Novel heterocyclic DPP-4 inhibitors for the treatment of type 2 diabetes}

Sutton $J M^{1}$, Clark $D E^{1}$, Dunsdon $S J^{1}$, Fenton $G^{1}$, Fillmore $A^{1}$, Harris $N V^{1}$, Higgs $C^{1}$, Hurley $C A^{1}$, Krintel $S L^{1}$, MacKenzie $R E^{1}$, Duttaroy $A^{2}$, Gangl $E^{2}$, Maniara $W^{2}$, Sedrani $R^{3}$, Namoto $K^{3}$, Ostermann $N^{3}$, Gerhartz $B^{3}$, Sirockin $F^{3}$, Trappe $J^{3}$, Hassiepen $U^{3}$, Baeschlin $D K^{3}$

${ }^{1}$ Argenta Discovery 2009 Ltd, Harlow, United Kingdom; ${ }^{2}$ Novartis Institutes for BioMedical Research, Cambridge, MA; and ${ }^{3}$ Novartis Institutes for BioMedical Research, Novartis Campus, Basel, Switzerland

Bioorg Med Chem Lett 2012; 22: 1464-8

Erratum in Bioorg Med Chem Lett 2012; 22: 2359

Newly discovered deazaxanthine-based DPP-4 inhibitors are shown to be potent $(\mathrm{IC}(50)<10 \mathrm{nM})$ and highly selective compared to other dipeptidyl peptidases. This report describes their synthesis, SAR, and initial efforts to improve their pharmacokinetic profile by modification of the deazaxanthine core. Optimization of compound $3 a$ led to the identification of compound (S)-4i, which displayed an improved in vitro and ADME profile. Further enhancements to the PK profile were achieved by changing from the deazahypoxanthine to the deazaxanthine template, leading to the development of compound $12 \mathrm{~g}$, which displayed good ex vivo DPP-4 inhibition and a superior PK profile in rats, which suggests the potential for once-daily dosing in humans.

\section{Inhibition of apical sodium-dependent bile acid transporter as a novel treatment for diabetes}

Chen $L^{1}$, Yao $X^{1}$, Young $A^{1}$, McNulty $J^{1}$, Anderson $D^{1}, \operatorname{Liu} Y^{1}$, Nystrom $C^{1}$, Croom $D^{1}$, Ross $S^{1}$, Collins $J^{2}$, Rajpal $D^{3}$, Hamlet $K^{1}$, Smith $C^{1}$, Gedulin $B^{4}$

Departments of ${ }^{1}$ Biology, ${ }^{2}$ Medicinal Chemistry, and ${ }^{3}$ Computational Biology, Quantitative Science, Metabolic Drug Discovery, GlaxoSmithKline, Inc., Research Triangle Park, NC; and ${ }^{4}$ Lumena Pharmaceuticals, Inc., San Diego, CA

\section{Am J Physiol Endocrinol Metab 2012; 302: E68-76}

Bile acids modulate metabolic activity. This study evaluated the effects of a potent apical sodium-dependent bile acid transporter (Asbt) inhibitor (264W94), which blocks intestinal absorption of bile acids, on glucose homeostasis in Zucker diabetic fatty (ZDF) rats. Following two weeks of oral 264W94, fecal bile acid concentrations had increased and nonfasting plasma total Glp-1 was elevated. Treatment with 
264W94 resulted in a significant decrease in $\mathrm{HbA} 1 \mathrm{c}$ and glucose and prevented the drop in insulin levels typically seen in ZDF rats in a dose-dependent manner. An oral glucose tolerance test revealed up to a two-fold increase in plasma total Glp-1 and a three-fold increase in insulin in ZDF rats treated with 264 W94 at doses sufficient to achieve glycemic control. Tissue mRNA analysis indicated a decrease in farnesoid $X$ receptor (Fxr) activation in the small intestines and the liver; however, coadministration of an Fxr agonist (GW4064) did not attenuate the 264W94-induced glucose-lowering effects. These results demonstrate that inhibition of Asbt increases bile acid levels in the distal intestine and promotes Glp-1 release. This approach may represent an effective new strategy for treating patients with type 2 diabetes mellitus.

\section{Comment}

The above six studies continue to explore new horizons in type 2 diabetes management, specifically SGLT-2 inhibitors, glucagon receptor antagonist, and DPP4 inhibitors. None of the SGLT-2 inhibitors have been approved by the FDA; however, one is pending FDA approval (canaglifozin). The long-term safety of these drugs is unknown, especially with Dapaglifozin. There was a disproportionate, though insignificant, increase in breast and bladder cancer. The future studies are ongoing to look at the effect of SGLT-2 inhibitions on other organs. There is also an attempt to study the effect of combined SGLT-2 and 1 inhibitors on glucose control. Some of the pharmaceutical companies are exploring the role of these drugs in type 1 diabetes. DPP4 inhibition has only shown modest response in glucose control in type $1 \mathrm{di}-$ abetes, especially in those individuals with positive $\mathrm{C}$ peptide levels. There are studies that are ongoing in early stages of exploring the role of SGLT-2 inhibitors in type 1 diabetes.

\section{Interleukin-1 receptor antagonist: a new therapy for type 2 diabetes mellitus}

\section{Akash MS, Shen Q, Rehman K, Chen S}

Institute of Pharmacology, Toxicology and Biochemical Pharmaceutics, College of Pharmaceutical Sciences, Zhejiang University, Hangzhou, Zhejiang Province, China

J Pharm Sci 2012; 101: 1647-58

A variety of complex mechanisms and pathways have a role in provoking low-grade local and systemic inflammation in $\beta$-cells of pancreatic islets and peripheral tissues, causing $\beta$ cell dysfunction and apoptosis, insulin resistance, and ultimately, overt type 2 diabetes mellitus (T2DM). Conventional antidiabetic agents have potential adverse effects, and there is a need for alternative treatments. Currently, Interleukin-1 receptor antagonist (IL-1Ra) is the only anti-inflammatory therapeutic modality that has been approved by U.S. Food and Drug Administration to treat patients with T2DM. We compared IL-1Ra with other anti-inflammatory agents and conventional antidiabetic agents. Although IL-1Ra has broadspectrum, anti-inflammatory activity, it has disadvantages due to its short half-life. To overcome this problem, we fused IL-1Ra with recombinant human serum albumin and ex- pressed it in Pichia pastoris. The bioactivity was confirmed in IL-1-induced A375.S2 apoptotic cells. We also formulated IL1Ra with Pluronic F-127-based thermo sensitive gel and investigated its in vitro characteristics related to duration of its therapeutic effects. Further studies are required to evaluate IL-1Ra's therapeutic efficacy in diabetes and diabetes-associated complications.

\section{Comment}

Interleukin-1 has been evaluated for its role in type 2 diabetes. New modulation in type 2 diabetes may have an effect in protecting beta-cell destruction by reducing inflammation. It is unknown if this effect is autoimmune in nature.

\section{A novel technique for the transplantation of pancreatic islets within a vascularized device into the greater omentum to achieve insulin independence}

Kriz $J^{1}$, Vilk $G^{2,3,4}$, Mazzuca $D M^{2,3,4}$, Toleikis $P M^{4}$, Foster PJ $J^{1}$, White DJG ${ }^{1,2,3,4}$

${ }^{1}$ Robarts Research Institute, University of Western Ontario, London, Ontario, Canada; ${ }^{2}$ Departments of Surgery and Pathology, University of Western Ontario, London, Ontario, Canada; ${ }^{3}$ Islet Transplantation Program, London Health Sciences Center, London, Ontario, Canada; and ${ }^{4}$ Sernova Corp., London, Ontario, Canada

Am J Surg 2012; 203: 793-7

\section{Background}

The greater omentum presents a promising location for islet transplantation due to its vascularization and blood flow. However, there is a need to provide a controlled and protected site for the islet cells within the omentum that would be suitable for use with donor islets and future stem cell technologies. This report describes a novel device with a subcutaneous delivery port that is implanted within the omentum and offers an environment for donor islets.

\section{Methods}

A prototype cell-pouch device was wrapped in the greater omentum of diabetic Lewis rats. An islet implantation port was exposed subcutaneously. After tissue growth throughout the device, islet isografts were implanted and long-term glucose control was evaluated.

\section{Results}

This technique resulted in long-term normal blood glucose levels in 7 of 10 diabetic rat recipients after minimal islet dose transplants. Histologic assessment confirmed collagen formation and vascularization within the device.

\section{Conclusions}

The implanted device provides a safe and efficacious environment to support the growth of transplanted pancreatic islet cells contained within a removable delivery device and administered as a cell therapy in a highly vascularized setting. 


\section{Comment}

There continues to be interest in improving implanted devices for pancreatic islets. This study was done in rats with the hope that it will be applicable in humans.

\section{Patients with type 2 diabetes initiating exenatide twice daily or insulin in clinical practice: CHOICE study}

Matthaei $S^{1}$, Reaney $M^{2}$, Mathieu $C^{3}$, Ostenson $C^{4}$, Krarup $T^{5}$, Guerci $B^{6}$, Kiljanski $J^{7}$, Petto $H^{8}$, Bruhn $D^{9}$, Theodorakis $M^{10}$

${ }^{1}$ Diabetes-Zentrum Quakenbr ck, Fachabteilung fur Diabetologie, Stoffwechsel und Endokrinologie am Christlichen Krankenhaus, Klinisches Diabeteszentrum der DDG, Akademisches Lehrkrankenhausder Medizinischen Hochschule Hannover, Quakenbruck, Germany; ${ }^{2}$ Eli Lilly, Windlesham, Surrey, United Kingdom; ${ }^{3}$ Department of Endocrinology, UZ Gasthuisberg, Leuven, Belgium; ${ }^{4}$ Department of Molecular Medicine and Surgery, Karolinska Institutet, Stockholm, Sweden; ${ }^{5}$ Department of Endocrinology I, Bispebjerg Hospital, Copenhagen, Denmark; ${ }^{6}$ Diabetologu, Nutrition, Metabolic Disroders, Brabois Hospital and Center of Clinical Investigation ILCV, CHU de Nancy, Vandoeuvre-Les-Nancy, France; ${ }^{7}$ Eli Lilly, Warsaw, Poland; ${ }^{8}$ Eli Lilly, Vienna, Austria; ${ }^{9}$ Eli Lilly, San Diego, CA; and ${ }^{10}$ Department of Clinical Therapeutics, University of Athens School of Medicine, Athens, Greece

Diabetes Ther 2012; 3: 6

\section{Introduction}

Initiating injectable therapy (CHOICE) is a European prospective, observational cohort study designed to assess time and factors associated with a significant change in therapy after patients with type 2 diabetes initiated their first injectable glucose-lowering therapy and to assess clinical outcomes over a 24-month period. Baseline data and factors associated with the injectable treatment regimen are reported.

\section{Methods}

Demographic, clinical, and healthcare resource-use data were collected at initiation of injectable therapy and analyzed using univariate tests between cohorts and multivariate logistic regression analysis for treatment.

\section{Results}

Overall, 1,177 patients initiated therapy with exenatide twice daily (bid), and 1,315 began insulin therapy. Patient recruitment was mainly by secondary-care physicians. Univariate analyses revealed statistically significant differences between the characteristics of patients who initiated exenatide bid and patients who initiated insulin. On multivariate analysis, higher body mass index [BMI; $5 \mathrm{~kg} / \mathrm{m}^{2}$ higher: odds ratio (OR) 2.10, 95\% confidence intervals $\{\mathrm{CI} ; 1.84-2.40\}$, lower glycated hemoglobin (HbA1c; $1 \%$ higher: OR $0.77,95 \% \mathrm{CI}$ $0.69-0.86$ ), and younger age (5 years older: OR $0.82,95 \% \mathrm{CI}$ $0.76-0.88)$ were the variables most strongly associated with increased probability of receiving exenatide bid $(p<0.0001)$. Patients initiating exenatide bid had a mean BMI of $35.3 \pm 6.5 \mathrm{~kg} / \mathrm{m}^{2}, \mathrm{HbA}(\mathrm{lc})$ of $8.4 \pm 1.4 \%$, and age of $58 \pm 10$ years; the comparative values for patients initiating insulin $(\mathrm{p}<0.0001)$ were $29.7 \pm 5.4 \mathrm{~kg} / \mathrm{m}^{2}, 9.2 \pm 1.9 \%$, and $64 \pm 11$ years, respectively. Other characteristics significantly associated with initiation of exenatide bid were "disinhibited eating" (Diabetes Health Profile-18), lower random blood glucose levels, less blood glucose self-monitoring, reduced lowdensity lipoprotein cholesterol levels, and receipt of diet/ exercise advice.

\section{Conclusions}

Patients who initiated exenatide bid were, on average, younger and more obese and had lower $\mathrm{HbA}(1 \mathrm{c})$ concentrations than those initiating insulin.

\section{Comment}

There are no guidelines for initiating injectable therapy (GLP analog or basal insulin) in subjects with type 2 diabetes who are failing oral treatment with metformin and/or sulfonylureas. It is entirely up to the physician when choosing the therapy for achieving target A1c in patients failing on orals. This study (CHOICE) further supports early treatment with incretins in lowering A1c values and an effective way to initiate treatment in younger obese subjects with diabetes. A much larger randomized control trial needs to be done that evaluates the effectiveness of the above two choices in initiating injectables in subjects with type 2 diabetes who are failing on oral treatment.

\section{New approaches for the treatment of diabetic macular oedema: recommendations by an expert panel}

Bandello $F^{1}$, Cunha-Vaz J ${ }^{2}$, Chong $N V^{3}$, Lang GE ${ }^{4}$, Massin $P^{5}$, Mitchell $P^{6}$, Porta $M^{7}$, Prünte $C^{8}$, Schlingemann $R^{9,10}$, Schmidt-Erfurth $U^{\mathbb{1}}$

${ }^{1}$ Department of Ophthalmology, University Vita-Salute, Scientific Institute San Raffaele, Milano, Italy; ${ }^{2}$ Association for Innovation and Biomedical Research on Light and Image, Coimbra, Portugal; ${ }^{3}$ Oxford Eye Hospital, University of Oxford, Oxford, United Kingdom; ${ }^{4}$ Department of Ophthalmology, University Eye Hospital, Ulm, Germany; ${ }^{5}$ Assistance Publique des Hôpitaux de Paris, Ophthalmology Department, Hôpital Lariboisière, Paris, France; ${ }^{6}$ Discipline of Ophthalmology, University of Sydney, Sydney, Australia; ${ }^{7}$ Department of Internal Medicine, University of Turin, Turin, Italy; ${ }^{8}$ Department of Ophthalmology and Optometry, Medical University of Vienna, Vienna, Austria and Vista Klinik, Binningen, Switzerland; ${ }^{9}$ Medical Retina Unit and Ocular Angiogenesis Group, Department of Ophthalmology, University of Amsterdam, Academic Medical Centre, Amsterdam, The Netherlands; ${ }^{10}$ Department of Clinical and Molecular Ophthalmogenetics, The Netherlands Institute for Neuroscience, Royal Netherlands Academy of Arts and Sciences (KNAW), Amsterdam, The Netherlands; and ${ }^{11}$ Department of Ophthalmology and Optometry, Medical University of Vienna, Vienna, Austria

Eye (Lond) 2012; 26: 485-93

The current standard therapy for patients with diabetic macular edema (DME) is focal/grid laser photocoagulation; however, it does not usually improve impaired vision, and many patients lose their vision despite laser therapy. The European Medicines Agency's recent approval of 
ranibizumab to treat visual impairment due to DME fulfills the previously unmet medical need for a treatment that can improve visual acuity (VA) in these patients. We reviewed one- and two-year clinical trial findings for ranibizumab therapy in DME to formulate evidence-based treatment recommendations. Patients with DME with or without visual impairment should be considered for ranibizumab treatment when the condition fulfills the Early Treatment Diabetic Retinopathy Study (ETDRS) criteria for clinically significant edema. For DME with centre involvement and associated vision loss due to DME, monthly ranibizumab monotherapy with treatment interruption and re-initiation based on VA stability is recommended. Laser therapy based on ETDRS guidelines is recommended for other forms of clinically significant DME without centre involvement or when no vision loss has occurred despite centre involvement. These recommendations are based on randomized controlled trials of one to two years duration. Therefore, the guidance may need updating as longterm ranibizumab data become available and clinical trial results for additional therapeutic agents become available.

\section{Comment}

Diabetic retinopathy, especially in type 1 diabetes, has significantly decreased, but in type 2 diabetes continues to increase (new NIH data recently released). This study uses ranibizumab in treatment of diabetic macular edema. A similar study in the past using avastin has been shown to improve visual loss deterioration.

\section{Early basal insulin therapy decreases new-onset diabetes after renal transplantation}

\section{Hecking $M^{1}$, Haidinger $M^{1}$, Döller $D^{1}$, Werzowa $J^{1}$, Tura $A^{2}$, Zhang $J^{3}$, Tekoglu $H^{4}$, Pleiner $J^{5}$, Wrba $T^{4}$, \\ Rasoul-Rockenschaub $S^{6}$, Mühlbacher $F^{6}$, Schmaldienst $S^{1}$, Druml $W^{1}$, Hörl $W H^{1}$, Krebs $M^{7}$, Wolzt $M^{8}$, Pacini $G^{2}$, Port $F K^{3}$, Säemann $M D^{1}$}

Departments of ${ }^{1}$ Nephrology, ${ }^{4}$ Informatics, ${ }^{5}$ Coordinating Center for Clinical Studies, ${ }^{6}$ Surgery, ${ }^{7}$ Endocrinology, and ${ }^{8}$ Clinical Pharmacology, Medical University of Vienna, Vienna, Austria; ${ }^{2}$ Metabolic Unit, Institute of Biomedical Engineering, National Research Council, Padova, Italy; and ${ }^{3}$ Arbor Research Collaborative for Health, Ann Arbor, MI

J Am Soc Nephrol 2012; 23: 739-49

New-onset diabetes after transplantation (NODAT) is an ongoing problem, and there are no effective interventions available to reduce this risk. The condition is associated with postoperative hyperglycemia and reduced patient and graft survival. In this one-year, proof-of-concept clinical trial, 50 renal transplant recipients were randomly assigned to receive immediate postoperative isophane insulin if they had evening blood glucose levels of $140 \mathrm{mg} / \mathrm{dl}$ (treatment group) or shortacting insulin and/or oral antidiabetic agents for blood glucose measurements of $180-250 \mathrm{mg} / \mathrm{dl}$ (standard-of-care/ control group). The study only included patients without a history of diabetes who received tacrolimus. By the third postoperative evening, all patients in the treatment group had blood glucose $140 \mathrm{mg} / \mathrm{dl}$ and were subsequently treated with basal insulin. During the first three weeks after transplanta- tion, the mean \pm SD daily insulin dosage was $17 \pm 11 \mathrm{IU} / \mathrm{d}$. Among controls, 23 of 25 (92\%) had blood glucose of $200 \mathrm{mg}$ / $\mathrm{dl}$, and 18 of $25(72 \%)$ received standard-of-care antihyperglycemic treatment. Asymptomatic hypoglycemia occurred five times in the treatment group and once in the control group. Throughout follow-up, the treatment group had $73 \%$ lower odds of NODAT (odds ratio, 0.27) than the control group, and hemoglobin A1c was on average $0.38 \%$ lower in the treatment group than in the control group. Twelve months after transplantation, all patients in the treatment group were insulin-independent, whereas 7 of $25(28 \%)$ patients in the control group required antidiabetic agents. The groups did not differ in terms of insulin sensitivity; however, the treatment group showed better beta-cell function throughout the oneyear follow-up. In conclusion, this study suggests that regimens that include basal insulin significantly reduce the odds for NODAT after renal transplantation, presumably via insulin-mediated protection of beta cells.

\section{Comment}

There continues to be an interest in initiating basal insulin therapy in early hyperglycemia for preventing diabetes. As we learned at the ADA, ORIGIN did not show any benefit on cardiovascular disease in basal insulin-treated group as compared to the standard therapy group. A larger sample size will be required to evaluate the true effect of early basal insulin treatment in post-renal transplant patients.

\section{Insulin degludec, an ultra-long-acting basal insulin, versus insulin glargine in basal-bolus treatment with mealtime insulin aspart in type 1 diabetes (BEGIN Basal-Bolus Type 1): a phase 3, randomised, open-label, treat-to-target non-inferiority trial}

Heller $S^{1}$, Buse $J^{2}$, Fisher $M^{3}$, Garg $S^{4}$, Marre $M^{5}$, Merker $L^{6}$, Renard $E^{7}$, Russell-Jones $D^{8,9}$, Philotheou $A^{10}$, Francisco $A M^{11}$, Pei $H^{12}$, Bode $B^{13}$, on behalf of the BEGIN Basal-Bolus Type 1 Trial Investigators

${ }^{1}$ University of Sheffield, Sheffield, United Kingdom; ${ }^{2}$ University of North Carolina School of Medicine, Chapel Hill, NC; ${ }^{3}$ Glasgow Royal Infirmary, Glasgow, United Kingdom; ${ }^{4}$ Barbara Davis Center for Childhood Diabetes, Aurora, CO; ${ }^{5}$ Hôpital BichatClaude Bernard, Assistance Publique des Hôpitaux de Paris, France; ${ }^{6}$ Diabetes und Nierenzentrum, Dormagen, Germany; ${ }^{7}$ Montpellier University Hospital, Montpellier, France; ${ }^{8}$ Royal Surrey County Hospital, Surrey, United Kingdom; ${ }^{9}$ University of Surrey, Surrey, United Kingdom; ${ }^{10}$ University of Cape Town Private Academic Hospital, Cape Town, South Africa; ${ }^{17}$ Novo Nordisk, Søborg, Denmark; ${ }^{12}$ Novo Nordisk, Princeton, NJ; and ${ }^{13}$ Atlanta Diabetes Associates, Atlanta, GA

Lancet 2012; 379: 1489-97

\section{Background}

In patients with type 1 diabetes mellitus, intensive basalbolus insulin therapy has been shown to improve glycemic control and reduce the risk of long-term complications. Insulin degludec is a new, ultra-long-acting form of basal insulin. This study compared the efficacy and safety of insulin 
degludec and insulin glargine, both administered once daily with mealtime insulin aspart, in basal-bolus therapy for patients with type 1 diabetes.

\section{Methods}

An open-label, treat-to-target, non-inferiority trial was undertaken at 79 sites (hospitals and centers) in six countries. The study subjects were adults (aged $\geq 18$ years) with type 1 diabetes (glycated hemoglobin [HbA1c] $\leq 10 \%$ [ $86 \mathrm{mmol} /$ mol]) who had been treated with basal-bolus insulin for at least one year. They were randomly assigned in a 3:1 ratio, using a computer-generated blocked allocation sequence, to receive insulin degludec or insulin glargine without stratification by use of a central interactive response system. The primary outcome was non-inferiority of degludec to glargine, assessed as a reduction in $\mathrm{HbA1c}$ after 52 weeks, with the intention-to-treat analysis. This trial is registered with ClinicalTrials.gov, number NCT00982228.

\section{Findings}

The trial included 629 participants: 472 were randomly assigned to receive insulin degludec and 157 insulin glargine. All participants were analyzed in their respective treatment groups. At one year, $\mathrm{HbA} 1 \mathrm{c}$ had fallen by $0.40 \%$ (SE 0.03 ) and $0.39 \%$ points (SE 0.07) with insulin degludec and insulin glargine, respectively (estimated treatment difference $-0.01 \%$ [95\% CI -0.14 to 0.11$] ; \mathrm{p}<0.0001$ for non-inferiority testing). Also at one year, $188(40 \%)$ and $67(43 \%)$ participants, respectively, achieved a target $\mathrm{HbA1c}$ of less than $7 \%(<53 \mathrm{mmol} /$ $\mathrm{mol}$ ). Rates of overall confirmed hypoglycemia (plasma glucose $<3.1 \mathrm{mmol} / \mathrm{L}$, characterized as severe) were similar in the insulin degludec and insulin glargine groups (42.54 vs. 40.18 episodes per patient-year of exposure; estimated rate ratio [degludec to glargine] 1.07 [0.89 to 1.28]; $\mathrm{p}=0 \cdot 48$ ). The rate of confirmed nocturnal hypoglycemia was $25 \%$ lower with degludec than with glargine (4.41 vs. 5.86 episodes per patientyear of exposure; 0.75 [0.59 to 0.96$] ; p=0 \cdot 021$ ). There were no substantial differences in serious adverse event rates (14 vs. 16 events per 100 patient-years of exposure) between the insulin degludec and insulin glargine groups.

\section{Interpretation}

Insulin degludec might be a useful form of basal insulin for patients with type 1 diabetes because it provides effective glycemic control while lowering the risk of nocturnal hypoglycemia, which is an important limiting factor in insulin therapy.

\section{Insulin degludec, an ultra-long-acting basal insulin, versus insulin glargine in basal-bolus treatment with mealtime insulin aspart in type 2 diabetes (BEGIN Basal-Bolus Type 2): a phase 3, randomised, open-label, treat-to-target non-inferiority trial}

Garber $A J^{1}$, King $A B^{2}$, Del Prato $S^{3}$, Sreenan $S^{4}$, Balci $M K^{5}$, Muñoz-Torres $M^{6}$, Rosenstock $J^{7}$, Endahl $L A^{8}$, Francisco $A M^{8}$, Hollander $P^{9}$, on behalf of the NN1250-3582 (BEGIN BB T2D) Trial Investigators

${ }^{1}$ Baylor College of Medicine, Houston, TX; ${ }^{2}$ Diabetes Care Center, Salinas, CA; ${ }^{3}$ University of Pisa, Pisa, Italy; ${ }^{4}$ Connolly Hospital, Dublin, Ireland; ${ }^{5}$ Akdeniz University School of Medicine, Antalya,
Turkey; ${ }^{6}$ University Hospital San Cecilio, Granada, Spain; ${ }^{7}$ Dallas Diabetes and Endocrine Center at Medical City, Dallas, TX; ${ }^{8}$ Novo Nordisk, Søborg, Denmark; and ${ }^{9}$ Baylor Medical Center, Dallas, TX

Lancet 2012; 379: 1498-507

\section{Background}

Ongoing loss of $\beta$-cell function is the hallmark of type 2 diabetes mellitus, resulting in deteriorating diabetes control. Conventional basal insulin therapy is not able to stop this loss of $\beta$-cell function. Insulin degludec is a new, ultra-long-acting basal insulin. The purpose of this study was to assess the efficacy and safety of insulin degludec compared with insulin glargine in patients with type 2 diabetes mellitus.

\section{Methods}

This 52-week, phase 3, open-label, treat-to-target, noninferiority trial was undertaken at 123 sites in 12 countries. It enrolled adults aged $\geq 18$ years with type 2 diabetes mellitus and a glycated hemoglobin ( $\mathrm{HbA} 1 \mathrm{c}$ ) of $7.0-10.0 \%$ after three months or more of any insulin regimen (with or without oral antidiabetic drugs). Eligible participants were randomly assigned in a 3:1 ratio to receive once-daily subcutaneous insulin degludec or glargine and were stratified by previous insulin regimen via a central interactive response system. Basal insulin was titrated to a target plasma glucose concentration of 3.9$<5.0 \mathrm{mmol} / \mathrm{L}$ self-measured before breakfast. The primary outcome was non-inferiority of degludec to glargine as determined by the change in $\mathrm{HbA} 1 \mathrm{c}$ from baseline to week 52 (noninferiority limit of $0.4 \%$ ) by ANOVA in the full analysis set. We assessed rates of hypoglycemia in all treated patients. This study is registered with ClinicalTrials.gov, number NCT00972283.

\section{Findings}

Of the total participant pool, 744 of $755(99 \%)$ randomly allocated degludec and 248 of 251 (99\%) assigned glargine were included in the full analysis set (mean age 58.9 years [SD 9.3]. Overall, $618(82 \%)$ and $211(84 \%)$ participants, respectively, completed the trial: diabetes duration 13.5 years [SD 7.3], $\mathrm{HbA} 1 \mathrm{c}$ 8.3\% [SD 0.8], and fasting plasma glucose $9.2 \mathrm{mmol} / \mathrm{L}$ [3.1]). After one year, HbA1c decreased by $1.1 \%$ in the degludec group and $1.2 \%$ in the glargine group (estimated treatment difference [degludec-glargine] $0.08 \%, 95 \%$ $\mathrm{CI}-0.05$ to 0.21 ), confirming non-inferiority. Rates of overall confirmed hypoglycemia (plasma glucose $<3.1 \mathrm{mmol} / \mathrm{L}$ or severe episodes requiring assistance) were lower with degludec than glargine (11.1 vs. 13.6 episodes per patient-year of exposure; estimated rate ratio $0.82,95 \%$ CI 0.69 to 0.99 ; $\mathrm{p}=0.0359)$, as were rates of confirmed nocturnal hypoglycemia (1.4 vs 1.8 episodes per patient-year of exposure; $0.75,0.58$ to $0.99 ; \mathrm{p}=0.0399$ ). No substantial difference was noted between the two groups for the rates of severe hypoglycemia (0.06 vs. 0.05 episodes per patient-year of exposure for degludec vs. glargine), but the numbers were too small for adequate assessment of differences. Rates of other adverse events did not differ between the groups.

\section{Interpretation}

A policy of suboptimum diabetes control to reduce the risk of hypoglycemia and its consequences in advanced type 2 
diabetes mellitus might be unwarranted with newer basal insulins such as degludec, which are associated with lower risks of hypoglycemia than insulin glargine.

\section{ABSTRACTS PRESENTED AT SCIENTIFIC MEETINGS}

\section{Insulin degludec does not increase antibody formation compared to insulin glargine: an evaluation of phase 3a clinical trials}

Vora $\mathrm{J}^{1}$, Hollander $\mathrm{P}^{2}$, Tamer $S C^{3}$, Johansen $T^{3}$, Bergenstal $\mathrm{RM}^{4}$

${ }^{1}$ Royal Liverpool University Hospitals, United Kingdom; ${ }^{2}$ Baylor University Medical Center, Dallas, TX; ${ }^{3}$ Novo Nordisk A/S, Soborg, Denmark; and ${ }^{4}$ International Diabetes Center at Park Nicollet, Minneapolis, MN

Diabetologia 2012; 55: S53-54

\section{Background and Aims}

Insulin degludec (IDeg) is an ultra-long-acting basal insulin analogue. It differs from insulin in that the threonine in position B30 of human insulin has been omitted and the lysine in position B29 has been coupled to hexadecanedioic acid via a glutamic acid spacer. IDeg forms soluble multi-hexamers on subcutaneous injection that have a stable and consistent glucose-lowering effect. While all insulin preparations cause some degree of antibody formation in humans, this has little or no effect on the efficacy or safety of currently marketed products. Here we present antibody data from all phase $3 a$ clinical trials in patients with type 1 or type 2 diabetes in which insulin antibody development associated with IDeg use was analyzed and compared to antibody formation related to insulin glargine (IGlar).

\section{Materials and Methods}

Insulin antibodies were measured in six randomized, controlled, open-label, treat-to-target trials ranging in duration from 26 to 52 weeks. Two of the trials included subjects with type 1 diabetes (IDeg: $n=801$; IGlar: $n=315$ ), and four trials were conducted in patients with type 2 diabetes (IDeg: $n=1734$; IGlar: $n=860$ ). In all trials, IDeg and IGlar were administered once daily. Insulin aspart was used as bolus insulin in the type 1 diabetes trials. Antibody levels (specific and cross-reactive to human insulin) were measured using specific radio-immunoassays and expressed as percent bound/total radioactivity $(\% \mathrm{~B} / \mathrm{T})$.

\section{Results}

Across all trials, few patients developed IDeg- and IGlarspecific antibodies and only at low levels. Furthermore, mean levels of antibodies that cross-reacted with human insulin remained low for both groups throughout the duration of the trials for subjects with type 1 diabetes $(10 \%-15 \% \mathrm{~B} / \mathrm{T})$ and type 2 diabetes $(<6 \% \mathrm{~B} / \mathrm{T})$. Spearman correlation coefficients were calculated on an individual trial basis for both IDeg and IGlar to investigate the influence of cross-reacting antibodies on $\mathrm{HbA} 1 \mathrm{c}$, change from baseline in $\mathrm{HbA} 1 \mathrm{c}$, and total daily insulin dose at end-of-trial. All correlation coefficients were low, indicating that there was no clinically relevant influence of insulin antibodies on $\mathrm{HbA} 1 \mathrm{c}$ or dose. Scatter plots revealed no difference in $\mathrm{HbA} 1 \mathrm{c}$ or total insulin dose at end-of-trial between patients having cross-reacting antibody levels of $>10 \% \mathrm{~B} / \mathrm{T}$ and those with levels $<10 \% \mathrm{~B} / \mathrm{T}$. The adverse-event profile in patients with an antibody titre of $>10 \% \mathrm{~B} / \mathrm{T}$ did not differ substantially from that of the overall trial population.

\section{Conclusion}

The results of this study indicate that immunogenic responses to long-term treatment with IDeg and IGlar are similar and low. Neither form of insulin had a clinically relevant impact on $\mathrm{HbA} 1 \mathrm{c}$ or total daily insulin dose.

\section{Comment}

The above three articles published in Lancet and Diabetologia report phase 3 registration studies data in type 1 and type 2 diabetes using insulin degludec. The first two studies illustrate that using degludec improves fasting blood glucose levels as compared to insulin glargine, with similar glucose control and insulin dose. However, the biggest advantage is in the reduction of hypoglycemia, especially nocturnal, in both type 1 and type 2 diabetes when compared with insulin glargine arm. It's also important to keep in mind that the differences in nocturnal hypoglycemia are small and thus will need to be seen if it can be replicated in real life. The new basal insulin, degludec, seems to be a promising agent for management of diabetes. As indicated above, it has only been approved in Japan and is pending approval in Europe and the United States. The third article highlights no excessive formation of antibodies to insulin degludec.

\section{Buccal spray insulin in subjects with impaired glucose tolerance: improvement in HbA1c is lost after 6 months washout therapy}

Palermo $A^{1}$, Napoli $N^{1}$, Maddaloni $E^{1}$, Lauria $A^{1}$, Soare $A^{1}$, Manfrini $S^{1}$, Altomare $M^{2}$, Leotta $S^{2}$, Pozzilli $P^{1}$

${ }^{1}$ Campus Bio Medico University of Rome, Italy; and ${ }^{2}$ Hospital "S.Pertini", Roma, Italy

Diabetologia 2012; 55: 53

\section{Background and Aims}

In the development of type 2 diabetes (TD2), affected individuals pass through a phase of impaired glucose tolerance (IGT). Defects in the action or secretion of insulin are the two major abnormalities that lead to glucose intolerance. Throughout the progression from normal glucose tolerance through IGT to diabetes, resistance to insulin gradually increases, whereas secretion of insulin gradually decreases. Glucose tolerance is assumed to remain normal as long as beta-cell secretion can compensate for the insulin resistance. IGT will develop only when insulin secretion fails to compensate fully for such resistance, resulting in postprandial hyperglycemia that is linked to an increased risk for cardiovascular disease, even though there is no progression to diabetes. Although many intervention trials have been conducted in patients with IGT, only one aimed to decrease postprandial hyperglycemia. Our previous proof-ofconcept study in subjects with IGT undergoing a prolonged IGTT demonstrated that treatment with 12 puffs of buccal spray insulin was followed by a significant (29.6\%) decrease in mean plasma glucose at $2 \mathrm{~h}$ and a $26.8 \%$ decrease at $3 \mathrm{~h}$. Based on 
these findings, a short trial with buccal spray insulin was designed.

\section{Materials and Methods}

This randomized controlled trial compared 6-month therapy with buccal spray insulin (12 puffs per meal) plus physical exercise and diet (treatment group $\mathrm{A}, \mathrm{n}=16, \mathrm{HbA} 1 \mathrm{c}$ at entry $6.2 \%+0.4$ ) with physical exercise and diet only (control group $\mathrm{B}, \mathrm{n}=16, \mathrm{HbA} 1 \mathrm{c}$ at entry $6.0 \%+0.3$ ) in patients with IGT. The primary endpoint was a difference in the reduction of $\mathrm{HbA} 1 \mathrm{c}$ of $0.3 \%$ at 6 months of treatment between the experimental versus control group. Secondary endpoints included antibody formations against insulin (IA), changes in body weight, number of hypoglycemic events during the treatment period, and the evaluation of metabolic control six months after the end of the treatment period. HbA1c levels, metabolic parameters, and insulin antibodies were measured at baseline, at 3 to 6 months, and after a 6-month washout period at the end of treatment.

\section{Results}

Subjects treated with buccal spray insulin achieved a significant reduction in $\mathrm{HbA1c}$ compared to the control group ( $\Delta$ HbA1c 0-6 months, negative (-) $0.3 \%$ vs. $+0.09 \% \mathrm{p}=0.002$ ). At 6 months after the end of treatment, in group A, HbAlc levels had increased from $5.8 \%+0.3$ to $6.1+0.5$, resulting in the loss of previously achieved improvement in metabolic control; $19 \%$ of the treated patients developed TD2 (vs. $6 \%$ in the control group). There was no significant difference in body weight, and no hypoglycemic or other adverse events were observed during the study period in either group. No insulin antibodies were detected in subjects with IGT treated with buccal spray insulin.

\section{Conclusion}

These results indicate that buccal spray insulin is an effective treatment compared to diet plus physical exercise in patients with IGT for reducing $\mathrm{HbA1c}$ without adverse effects. However, the beneficial effects of buccal spray insulin is lost within few months of suspension of treatment. A larger trial is required to demonstrate the long-term effects of buccal spray insulin and its ability to prevent TD2 in subjects with IGT.

\section{Comment}

Buccal insulin spray has been evaluated in the past decade by many investigators and with several publications and presentations at different international diabetes meetings. Most of the data from the previous studies have been difficult to be replicated and thus a proper phase 2 or phase 3 study has never been done in the United States or in Europe. This study evaluates the use of buccal insulin spray in impaired glucose tolerance subjects. If this effect is biological and due to absorption of insulin through the buccal mucosa, a proper PK/PD study and a much larger clinical study needs to be performed by independent investigators. To the best of my knowledge, this product is approved for use in Ecuador for management of type 2 diabetes; however, its exact role in diabetes management still needs to be established.
Reduced postprandial glucose excursion in type 2 diabetic subjects using the InsuPad device

Bitton $G^{1}$, Raz $I^{2}$, Pfutzner $A^{3}$, Feldman $D^{1}$, Alon $T^{1}$, Liviatan $L^{1}$, Nagar $R^{1}$

${ }^{1}$ Insuline Medical Ltd., Petach Tikva, Israel; ${ }^{2}$ The Diabetes Center, Hadassah Hebrew University School of Medicine, Jerusalem, Israel; and ${ }^{3} I K F E-I n s t i t u t e$ for Clinical Research and Development, Mainz, Germany

Diabetologia 2012; 55: S54

\section{Objective}

The pharmacodynamic (PD) and pharmacokinetic (PK) profiles of current insulin analogs are still suboptimal compared to normal physiology. Among other effects, this results in large postprandial blood glucose excursions in patients with insulin-dependent diabetes (IDDS). InsuLine Medical has developed a technology to accelerate the insulin PK profile by applying local, controlled heat to the insulin delivery site. This heat increases local blood flow, which can induce faster insulin clearance from the injection site. This can improve glycemic control. In this study, we have evaluated the effects of the InsuPad device on postprandial glucose during meal tolerance testing.

\section{Methods}

A meal tolerance test protocol was conducted in subjects with type II diabetes who use insulin injections with rapidacting insulin analogs. The participants injected $0.2 \mathrm{U} / \mathrm{kg}$ before consuming a standardized liquid meal (Ensure Plus [Abbott], $474 \mathrm{ml}$, carbohydrate $100 \mathrm{~g}$, protein $26 \mathrm{~g}$, fat $23 \mathrm{~g}$ ). Blood samples for glucose and insulin measurements were taken from a venous line throughout the study. The study was repeated twice, once with (test) and once without (control) the use of the InsuPad device.

\section{Results}

Ten subjects with type II diabetes participated in the study, aged 51 to 68 years [mean 61.4 years], HbA1c $7-9.8 \%$ [mean $8.1 \%$ ], BMI $23-35 \mathrm{~kg} / \mathrm{m}^{2}$ [mean $26.5 \mathrm{~kg} / \mathrm{m}^{2}$ ], and mean diabetes duration 24.5 years. Glucose excursion within 250 minutes after meal start (G-AUC0-250) decreased when the InsuPad device was used $(23.9 \pm 43.4 \mathrm{mg} / \mathrm{dL} / \mathrm{H}$ with the device compared to $63 \pm 36 \mathrm{mg} / \mathrm{dL} / \mathrm{H}$ without the device $\mathrm{p}=0.08$ ). Glucose excursion at 120 minutes post meal (G120) also decreased when the InsuPad device was used $(28 \pm 65.3 \mathrm{mg} / \mathrm{dl}$ with the device $v s$. $96.4 \pm 67.5 \mathrm{mg} / \mathrm{dl}$ without the device $\mathrm{p}=0.033$ ). Maximal glucose excursion (Gmax) was also reduced with the InsuPad device $(79.8 \pm 52.2 \mathrm{mg} / \mathrm{dl}$ vs. $136.8 \pm 53.6 \mathrm{mg} / \mathrm{dl} \mathrm{p}=0.053)$

\section{Discussion and Conclusion}

These results demonstrate a mean reduction of more than $60 \%$ in postprandial glucose excursion during the 6-hour period after the start of a meal. Such a significant decrease, if repeated in daily life conditions, could significantly improve glycemic control and reduce HBA1c levels in people with type II diabetes. Future plans aim to increase the number of subjects participating in the study and to repeat the study under daily life conditions. 


\section{Improved pharmacokinetic and pharmacodynamic profile of insulin analogues using InsuPatch, a local heating device}

Landau $Z^{1}$, Feldman $D^{1}$, Shusterman $A^{1}$, Wainstein $J^{1}$, Klonoff $D^{2}, \operatorname{Nyberg} I^{2}$, Lender $D^{3}$, Mosenzon $O^{3}$, Raz $I^{3}$

${ }^{1}$ Diabetes Unit, E. Wolfson Medical Center, Holon, Israel; ${ }^{2}$ Diabetes Research Institute, Mills-Peninsula Health Services, San Mateo, CA; and ${ }^{3}$ Diabetes Unit, Hadassah University Hospital, Jerusalem, Israel

Diabetologia 2012; 55: S54

\section{Background and Aims}

The pharmacological profile of rapid-acting insulin analogs does not yet sufficiently mimic the profile of endogenous insulin release. Studies suggest that heating the insulin injection site may accelerate insulin absorption by increasing local blood flow. InsuPatch is a local heating device intended to accelerate subcutaneous insulin absorption and activity, when used with an insulin pump, by warming the infusion site. The device elevates the temperature of the tissue surrounding the infusion site to $38.5^{\circ} \mathrm{C}$ for 30 minutes following the administration of an insulin bolus. The aim of this study was to compare the pharmacodynamic and pharmacokinetic profiles of subcutaneously administered rapid-acting insulin analogs delivered by insulin pump with and without InsuPatch.

\section{Materials and Methods}

Euglycemic glucose clamp tests were performed in 56 subjects with type 1 diabetes who used an insulin pump (mean age $33.4 \pm 13.5$ years, 23 female, HbA1c $7.75 \pm 0.85 \%$ ) after administration of 0.15 units $/ \mathrm{kg}$ body wt of short-acting insulin analogs, with or without InsuPatch, using a randomized crossover design. The 55 subjects who participated in the study each underwent three clamp procedures: two clamp studies with the InsuPatch device on day 1 and on day 3 of a three-day infusion set cycle (test), and one clamp study without the device on day 1 of the infusion set cycle (control). The second clamp study with InsuPatch on day 3 was performed to examine whether there was any effect of increasing duration of infusion site use on the absorption and time-action profile of short-acting insulin analogs.

\section{Results}

The area under the insulin concentration curve during the initial 60 minutes (insulin AUC0-60) increased by $29.7 \pm 7 \%$ with the use of InsuPatch compared to the control procedure on day $1(p=0.012)$. The insulin AUC0-60 was found to increase by $27.9 \pm 7 \%$ on day 3 with InsuPatch compared to day 1 with InsuPatch $(p<0.05)$. Data are presented for days 1 and 3 under test and control conditions for maximal insulin concentration (C-INSmax), time to maximum glucose infusion rate (T-GIRmax), and maximal glucose infusion rate (GIR$\max )$. The results indicate that the device had a greater effect in the subgroup of participants with $\mathrm{HbA} 1 \mathrm{C}$ concentrations above the mean $\mathrm{HbA} 1 \mathrm{C}(7.76 \%, \mathrm{n}=26)$; the relative increase in AUC0-60 was $45 \%(\mathrm{p}<0.05)$. We report the same incidence of local irritations with and without the heating device (on day 1 and day 3).

\section{Conclusion}

Administration of short-acting insulin analogs with InsuPatch, a local heating device, to subjects with type 1 diabetes who use an insulin pump enhances insulin absorption and reduces the duration of glucose-lowering action. This approach might enable better meal-related insulin coverage and thereby permit better control of postprandial glycemia.

\section{Comment}

The above two studies have made an attempt to increase the absorption of rapid-acting insulin analogues by local heating with a hope of rapid onset of action. The temperatures used in the two studies were different $\left(38^{\circ}\right.$ and $39.5^{\circ} \mathrm{C}$ ). The sample size is small and the devices used are slightly different in the two studies. A much larger study needs to be performed to see the efficacy of using local heating pads to increase insulin absorption. However, there are many other ways to achieve a faster action of rapid-acting insulin analogues, for example, adding hyluronidase with rapid-acting insulin and adding vitamin $\mathrm{D}$ to aspart, etc. The development of ultra-rapid-acting insulin is important if we are going to mimic normal physiology and blunt postprandial glucose excursions significantly and above all develop a true closed-loop device that mimics physiology.

\section{In vitro characterisation of novel basal insulin LY2605541: reduced mitogenicity and IGF-IR binding}

Owens RA, Lockwood JF, Dunbar JD, Zhang C, Ruan X, Kahl SD, Qian HR, Beals JM

Diabetologia 2012; 55: S55

\section{Background and Aims}

LY2605541 (LY) is insulin lispro with a $20 \mathrm{kDa}$ polyethylene glycol (PEG) moiety covalently attached to lysine B28. The design of LY is intended to create a large hydrodynamic diameter to reduce subcutaneous absorption and plasma clearance. This should result in prolonged duration of action, which is desired for a basal insulin. Given this design, it is critical to understand the in vitro biological properties of LY in terms of function, selectivity, and mitogenicity.

\section{Materials and Methods}

We dissolved zinc-free biosynthetic human insulin, insulin lispro AspB10 insulin, and LY in PBS, pH 7.4. Insulin detemir and insulin glargine were dissolved in diluents and treated with Chelex 100 resin to remove zinc from the formulations. They were then dialyzed into PBS or citrate buffer, $\mathrm{pH} 4$, respectively. A competitive radioligand scintillation proximity binding assay (SPA) was designed and was carried out using albumin-free NP-40 binding buffer and 293HEK cell membranes that over-expressed either the human insulin receptor (IR) or the human insulin-like growth factor-1 receptor (IGF1R). A tyrosine phosphorylation ELISA was used to determine the functional activity in IR over-expressing cells. We also evaluated de novo lipogenesis from [U-14C]-glucose using differentiated 3T3-L1 adipocytes. Since insulin and IGF-1 act through their cognate receptors to increase DNA synthesis 
and cell proliferation, two cell lines-a human osteosarcoma cell line (SAOS2) that expresses more IGF-1R than IR, and a rat hepatoma cell line (H4IIe) that expresses only rat IR-were selected as cellular models for in vitro mitogenicity studies based on assessment of methyl-3H-thymidine incorporation into the cellular DNA of SAOS-2 cells, and proliferation (Cell Titer, Promega) in the H4IIe cells. The data were analyzed using four-parameter nonlinear regression (Activity Base, IDBS). We report the results in the form of the dose required to achieve a half-maximal response (EC50) compared to the BHI reference standard. The affinity constant $(\mathrm{Ki})$ was calculated from the IC50 value using the Cheng-Prusoff equation. All results are the geometric mean $\pm S E M, n$.

\section{Results}

LY shows a 17-fold lower-binding affinity than insulin lispro at IR $(7.1 \pm 2.5 \mathrm{nM} ; \mathrm{n}=3$ and $0.41 \pm 0.02 \mathrm{nM} ; \mathrm{n}=3$, respectively) and a $>32$-fold lower-binding affinity than insulin lispro at IGF-1R (>1500 nM; $n=3$ and $48 \pm 4 n M ; n=3$, respectively). Based on these receptor-binding results, we conclude that LY is more selective than insulin lispro for IR vs. IGF-1R (>210-fold vs. 110-fold, respectively). LY is an effective and potent agonist for IR auto-phosphorylation (EC50= $20 \pm 2 \mathrm{nM} ; \mathrm{n}=3$ ) and de novo lipogenesis (EC50=16 $\pm 4 \mathrm{nM}$; $n=6)$. LY shows reduced mitogenic potency compared to insulin lispro in both the IGF-1R-dominant and the IR-dominant cell line (78-fold less and 15-fold less, respectively). In comparison to other forms of insulin, LY has similar in vitro characteristics related to signaling mediated through IR as compared to insulin lispro, insulin detemir, and insulin glargine. LY shows improved selectivity for IGF-1R signaling as compared to insulin lispro and insulin glargine.

\section{Conclusion}

Despite having a reduced binding affinity following PEGylation, LY maintained all of the in vitro characteristics of its parent, insulin lispro, and achieved all of the metabolic and mitogenic characteristics desired in a long-acting insulin.

\section{Comment}

Another way to prolong the action of insulin is to pegylate the insulin molecule to deliver a true basal insulin. The first of this kind is being evaluated by Eli Lilly \& Company, where insulin Lispro has been pegylated to make a large molecule. It has a long T-1/2 and duration of action lasting up to 72 hours. Multiple studies in both type 1 and type 2 diabetes (phase 2 ) were presented at the ADA this year with a promise of an ideal basal insulin. The above study highlights the safety of new insulin and several Phase 2 studies are ongoing in the United States, Europe, and many other parts of the world. I'm sure next year's yearbook will see much of that data presented, either during the conference or in the form of manuscripts.

\section{Summary}

There are many oral and injectable treatment options being evaluated for diabetes management. These include SGLT 1 or 2 or dual inhibitors, newer DPP-IV inhibitors, longer acting GLP-1 analogs (once a day, a week, or even implantable lasting for several months), and glucokinase activators. Several new basal insulins (degludec, now approved in Japan and still not approved in Europe or the United States, and pegylated insulin Lispro) are being investigated since the use of basal insulin as an early therapeutic option in Type 2 diabetes is accepted now by more providers across the globe.

Another approach is to use currently available rapid-acting insulin in a patch pump with continuous delivery of basal insulin, as has been discussed in the device chapter. The currently available rapid-acting insulins are not that rapid in onset of action, and thus, patients end up having significant postprandial glucose excursions if not injected 15-30 minutes before meals, which is not practical nor is it followed by the patients. This is contrary to what is marketed to the providers. Newer prandial insulins that might mimic physiology are being evaluated. Those include hylenex-hyluronidase + lispro or aspart, FI-ASP attaching vitamin E (Nicotinamide) to aspart for facilitating rapid absorption, or using local heat to improve insulin absorption. Last but not the least, there is emerging interest in making "SMART" insulin that responds to patients' needs as it relates to basal or prandial insulin. These insulins have chemical sensors as a part of a specially engineered molecule, which can be injected once a day and will meet the demands of the patient for both basal and bolus insulin appropriately.

This is an exciting time to be involved with diabetes care, especially when we know that global interest in the disease is increasing, with significant interest from pharmaceutical companies to develop new molecules that impact diabetes management.

\section{Author Disclosure Statement}

S.K.G. declares that no competing financial interests exist. 